\title{
Magnetospheric Accretions and the Inner Winds of Classical T Tauri Stars
}

\author{
Ryuichi Kurosawa $^{1}$ and M. M. Romanova ${ }^{2}$ \\ ${ }^{1}$ Max-Planck-Institut für Radioastronomie, \\ Auf dem Hügel, 69, 53212 Bonn, Germany \\ email: kurosawa@mpifr-bonn.mpg.de \\ ${ }^{2}$ Department of Astronomy, Cornell University, \\ Ithaca, NY 14853-6801, USA \\ email: romanova@astro.cornell.edu
}

\begin{abstract}
Recent spectropolarimetric observations suggest that young low-mass stars such as classical T Tauri stars (CTTSs) possess relatively strong $(\sim \mathrm{kG})$ magnetic field. This supports a scenario in which the final accretion onto the stellar surface proceeds through a magnetosphere, and the winds are formed in magnetohydrodynamics (MHD) processes. We examine recent numerical simulations of magnetospheric accretions via an inclined dipole and a complex magnetic fields. The difference between a stable accretion regime, in which accretion occurs in ordered funnel streams, and an unstable regime, in which gas penetrates through the magnetosphere in several unstable streams due to the magnetic Rayleigh-Taylor instability, will be discussed. We describe how MHD simulation results can be used in separate radiative transfer (RT) models to predict observable quantiles such as line profiles and light curves. The plausibility of the accretion flows and outflows predicted by MHD simulations (via RT models) can be tested against observations. We also address the issue of outflows/winds that arise from the innermost part of CTTSs. First, we discuss the line formations in a simple disk wind and a stellar wind models. We then discuss the formation of the conically shaped magnetically driven outflow that arises from the disk-magnetosphere boundary when the magnetosphere is compressed into an X-type configuration.
\end{abstract}

Keywords. stars: low-mass, stars: magnetic fields, stars: winds, outflows, stars: pre-mainsequence, stars: variables: other, MHD, radiative transfer, line: formation, line: profiles

\section{Introduction}

In the standard picture of accretions in classical T Tauri stars (CTTSs), the stellar magnetic fields are assumed to be strong enough to truncate the accretion disks near the star-disk corotation radii (e.g., Camenzind 1990; Koenigl 1991). The matter originating in the vicinity of the disk truncation radius flows along the magnetic field lines to the stellar surface. The kinetic energy of the infalling matter is converted into the thermal energy of the plasma in the shock regions above the photosphere. This general picture of magnetospheric accretions successfully explains some of the important observational aspects of CTTSs, e.g., the UV-optical continuum excess (e.g. Calvet \& Gullbring 1998) and the inverse P-Cygni profiles (e.g. Hartmann et al. 1994; Muzerolle et al. 2001). In the earlier magnetospheric accretion models (e.g. Koenigl 1991; Hartmann et al. 1994), the flows are often assumed to be steady and axisymmetric, as in the original models for accreting neutron stars (e.g. Ghosh et al. 1977) . While these axisymmetric models agree with some of the observational phenomena as mentioned above, they are not applicable to many other observational aspects. For example, (1) some CTTSs are known to exhibit complex line and continuum variability (e.g. Johns \& Basri 1995; Petrov et al. 1996), 
and (2) recent time-series spectropolarimetric (Zeeman-Doppler Imaging) observations (Donati et al. 2007, 2011; Jardine et al. 2008; Gregory et al. 2008; Hussain et al. 2009) have revealed that some of CTTSs have complex stellar magnetic fields. Clearly, these observational aspects (complex variability and accretion geometry) cannot be explained by simple axisymmetric models. To address the issues of the complex variablity and nonaxisymmetry of CTTSs, we will use three-dimensional (3-D) radiative transfer models of time-series line profiles using the results of 3-D magnetohydrodynamics (MHD) simulations of the accretion onto CTTSs through inclined magnetic multipoles. The results are presented in Sec. 2

Not only the accretion flows, but also the outflows/winds in CTTSs are most likely shaped by magnetic fields. The formation of the outflow itself likely occurs in MHD processes in which open magnetic fields are anchored to a star, an accretion disk or both (e.g., Ferreira et al. 2006). Observationally, there are ample of spectroscopic evidences that rather high velocity winds/outflows originate from the innermost part of the stardisk systems, e.g., the blueshifted absorption component in strong optical and near-UV lines such as H $\alpha$, Na I D, Ca II H\&K (e.g., Reipurth et al. 1996; Alencar \& Basri 2000; Ardila et al. 2002). In addition, the near-infrared He I $\lambda 10830$ line has been recognized as a robust wind diagnostic line by e.g. Takami et al. (2002), Edwards et al. (2003), Edwards et al. (2006). In particular, Edwards et al. (2006) showed that the line is very sensitive to the presence of the wind, and about 70 per cent of CTTSs exhibit a blueshifted absorption (below continuum) in He I $\lambda 10830$ while only about 10 per cent of CTTSs show a similar type of absorption component in $\mathrm{H} \alpha$ (Reipurth et al. 1996). Edwards et al. (2006) and Kwan et al. (2007) suggested that the blueshifted absorption component in He I $\lambda 10830$ profiles is cased by a stellar wind in about 40 per cent, and by a disc wind in about 30 per cent of the samples in Edwards et al. (2006). In Sec. 3, we explore how the radiative transfer models of He I $\lambda 10830$ can be used to probe the origin of the inner winds of CTTSs. We will also test the plausibility of the conically shaped magnetically driven wind ("the conical wind") solution found in the MHD simulations of Romanova et al. (2009).

\section{Non-Axisymmetric Accretion Models and Complex Variablity}

In this section, we examine the types of variability expected from (1) steady nonaxisymmetric accretion flows (Sec. 2.1), and (2) unsteady non-axisymmetric accretion flows (Sec. 2.2).

\subsection{Rotationally Induced Modulations Caused by Steady Accretion Flows}

A set of radiative transfer models which simulate the rotationally induced line variability arising from a complex circumstellar environment of CTTSs was presented by Kurosawa et al. (2008) (see also Symington et al. 2005; Kurosawa et al. 2005). The results of the 3-D MHD simulations from Romanova et al. (2003) and Romanova et al. (2004), who considered accretion onto a CTTS with a misaligned dipole magnetic axis with respect to the rotational axis, were used in a separate $3-\mathrm{D}$ radiative transfer model (e.g., Harries 2000; Kurosawa et al. 2006) to predict observed line profiles (e.g., $\mathrm{H} \beta, \mathrm{Pa} \beta$ and $\mathrm{Br} \gamma$ ) as a function of rotational phases. The accretion in those MHD models are in a steady state, and the accretion mainly proceeds in two funnel flows, i.e., one lands on the upper and the other on the lower hemispheres. In this study, the general dependencies of line variability on inclination angles $(i)$ and magnetic axis misalignment angles $(\Theta)$ were examined.

Using the same method, we now examine the accretion flows with more complex magnetic field configurations (with a combination of an inclined dipole and an octupole 

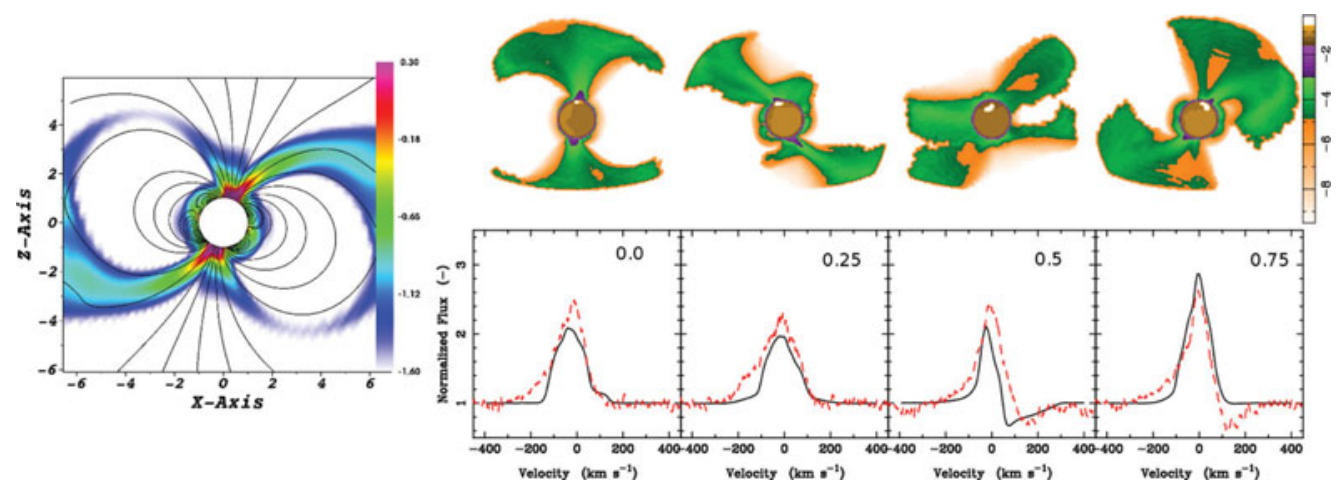

Figure 1. Left panel: A cross section of the density from the 3-D MHD simulations of accretion onto the CTTS V2129 Oph. The density is shown in logarithmic scale and in arbitrary units. The solid lines represent sample magnetic field (a combination of a dipole and an octupole). Right panels: The radiative transfer models computed for the accretion flows from the MHD simulation. Upper panels show the $\mathrm{H} \delta$ emission maps at four different rotation phases of the star $(0.0,0.25,0.5$ and 0.7 from left to right). Lower panels show the corresponding line profiles models of $\mathrm{H} \delta$ (solid) along with the data obtained by ESPaDOnS (dashed). The models and data are from Alencar et al. (2012).

components), similar to those in Romanova et al. (2011) and Long et al. (2011). In particular, the accretion flows around the CTTS V2129 Oph (K5) were modeled by the MHD simulations in which the information from the surface magnetic field map (by the Zeeman-Doppler imaging technique) obtained by Donati et al. (2011) was incorporated (see Alencar et al. 2012). For example, the observation by Donati et al. (2011) suggests that the inclinations of the magnetic dipole and the octupole are about $15^{\circ}$ and $25^{\circ}$ with respective to the rotation axis, and the octupole axis is about 0.1 ahead of the dipole in the rotational phase. A single time-slice of the MHD simulations was used to predict the dependency of the observed line profiles on the rotational phase by using the 3-D radiative transfer model mentioned earlier. The results are shown in Fig. 1. As one can see from this figure, near the stellar surface, the octupole component dominates and it redirect the accretion funnels to a higher latitude. On the other hand, the dipole component dominates in larger scales, and it interact with the innermost part of the accretion disk. The line profile models for $\mathrm{H} \beta$ are compared with the observations obtained by ESPaDOnS at CFHT (Alencar et al. 2012) at four different rotational phases (0.0, 0.25, 0.5 and 0.75 ). The figure shows that our model agrees with the time-series observed line profiles very well. More detailed comparisons of the model with observations can be found in Alencar et al. (2012).

\subsection{Irregular Variability Caused by Accretion in an Unstable Regime}

In the previous section (Sec. 2.1), we considered the cases in which the accretion flows are steady even though the flows themselves are non-axisymmetric. Here, we examine the unsteady accretion flows caused by the magnetic Rayleigh-Taylor (R-T) instability, which occurs at the interface between an accretion disk and a stellar magnetosphere. The instability tends to appear in a system with a relatively slowly rotating magnetosphere, a high mass-accretion rate and a small misalignment angle for the magnetic poles. The detail theoretical studies on the conditions for instability have been presented by e.g., Arons \& Lea (1976); Spruit \& Taam (1993); Li \& Narayan (2004). More recently, the global three-dimensional (3D) magnetohydrodynamic (MHD) simulations by e.g., Romanova et al. (2008); Kulkarni \& Romanova (2008, 2009) have shown that the R-T instability 

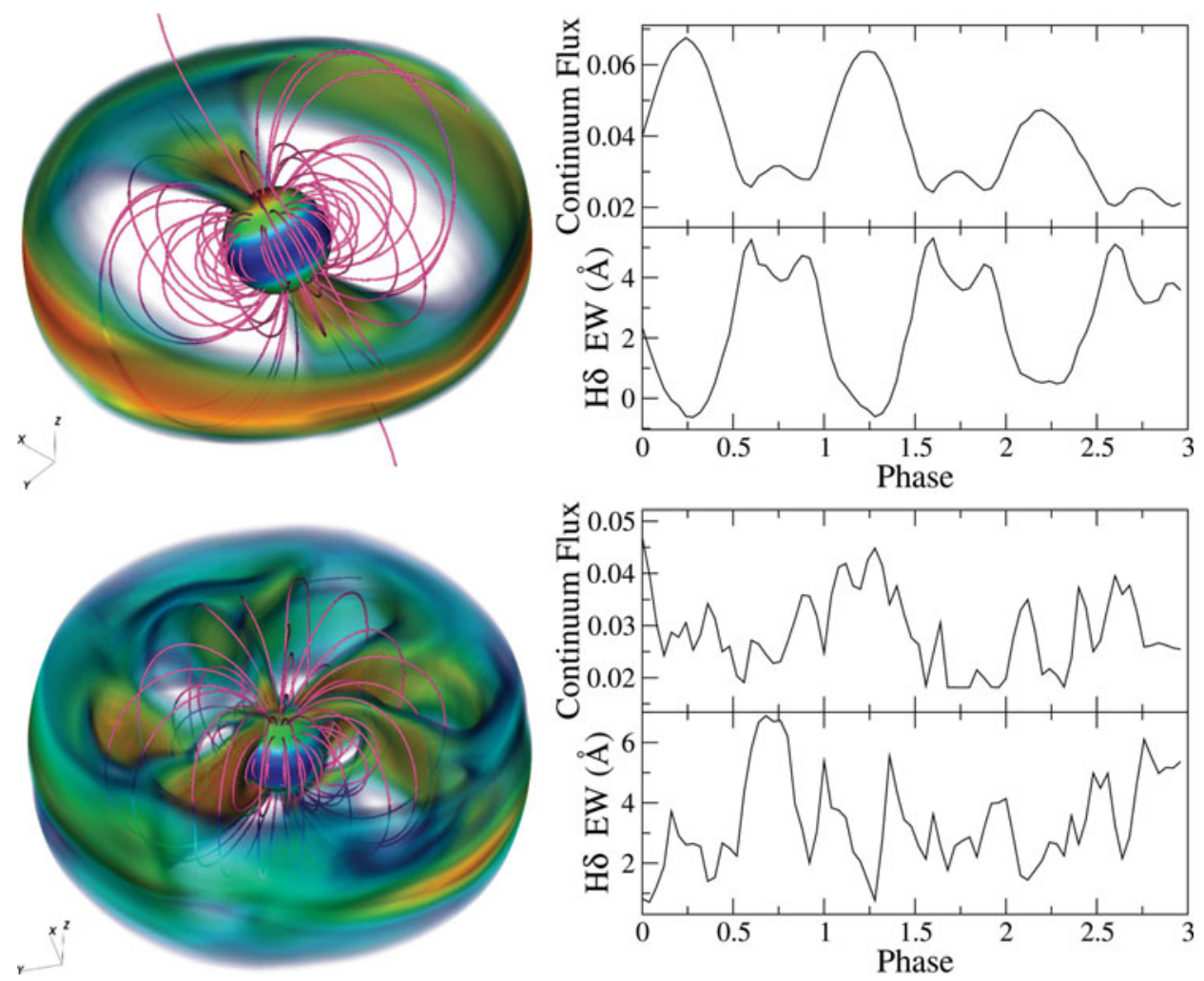

Figure 2. A comparison of accretions onto a CTTS in a stable (upper panels) and an unstable (lower panes) regimes. While the density distributions (volume renderings; in logarithmic scales; in arbitrary units) from the 3D MHD simulations are shown in the left panels, the radiative transfer models for the continuum flux and $\mathrm{H} \delta$ line equivalent widths plotted as a function of rotation phases are shown on the right panels. The continuum flux are evaluated near $\mathrm{H} \delta$. The figures are from Kurosawa \& Romanova (2013).

induces accretions in multiple (a few to several) unstable vertically elongated streams or 'tongues' which penetrate the magnetosphere (the lower left panel in Fig. 2). They found that the corresponding time-scale of the variability induced by the instability is typically a few times smaller than the rotation period of a star. Here, we examine the fundamental differences in the observational properties of the accretions in the stable and unstable regimes.

The results of global 3-D MHD simulations of matter flows in both stable and unstable accretion regimes are used in Kurosawa \& Romanova (2013) to calculate time-dependent hydrogen line profiles and study their variability behaviors (see Fig. 2). In the stable regime, some hydrogen lines (e.g., $\mathrm{H} \beta, \mathrm{H} \gamma, \mathrm{H} \delta, \mathrm{Pa} \beta$ and $\mathrm{Br} \gamma$ ) show a redshifted absorption component only during a fraction of a stellar rotation period, and its occurrence is periodic. However, in the unstable regime, the redshifted absorption component is present rather persistently during a whole stellar rotation cycle, and its strength varies non-periodically. In the stable regime, an ordered accretion funnel stream passes across the line of sight to an observer only once per stellar rotation period while in the unstable regime, several accreting streams/tongues, which are formed randomly, pass across the line of sight to an observer. The latter results in the quasi-stationarity appearance of the redshifted absorption despite the strongly unstable nature of the accretion. In the unstable regime, multiple hot spots form on the surface of the star, producing the 

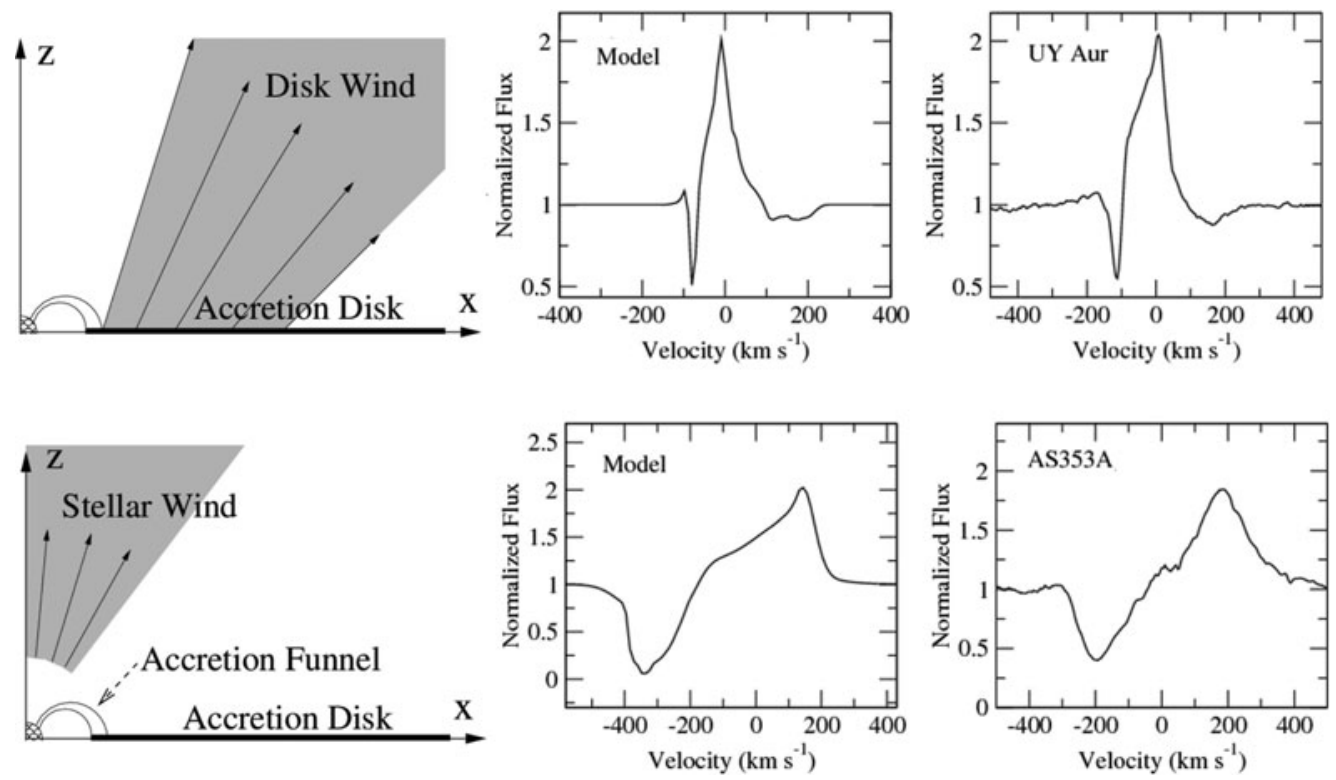

Figure 3. Simple axisymmetric kinematic disk wind (upper panels) and stellar wind (lower panels) models that are combined a dipolar magnetospheric accretion. The left panels show the flow configurations. The corresponding line profile models of He I $\lambda 10830$ are shown in the middle panels. The inclination angles $(i)$ used for computing the line profiles are $i=48^{\circ}$ and $10^{\circ}$ for the models with a disk wind and a stellar wind, respectively. The observed He I $\lambda 10830$ line profiles for the CTTSs UY Aur and AS 353 A (from Edwards et al. 2006) are shown in the right panels, for qualitative comparisons. The figures are from Kurosawa et al. (2011).

stochastic light curve with several peaks per rotation period. Interestingly, such irregular light-curves are frequently observed in CTTSs (e.g. Herbst et al. 1994; Rucinski et al. 2008; Alencar et al. 2010). Note that irregular light curves are found in about 39 per cent of the CTTS samples in Alencar et al. (2010). No clear periodicity in the line variability is found in many CTTSs. This study suggests a CTTS that exhibits a stochastic light curve and a stochastic line variability, with a rather persistent redshifted absorption component, may be accreting in the unstable accretion regime.

\section{The Inner Winds of Classical T Tauri Stars}

Understanding the origin of an outflow, whether it is a stellar wind, the X-wind (Shu et al. 1994), the conical wind (Romanova et al. 2009) or disk wind, is important as it is closely related to the angular momentum evolution of young stellar objects. Here, we demonstrate how the line profile models can be used to probe the origin of the inner winds.

\subsection{Simple Kinematics Wind Models}

As briefly mentioned in Sec. 1, Edwards et al. (2006) have demonstrated a robustness of the near-infrared He I $\lambda 10830$ line for probing the inner winds of CTTSs. In particular, they have classified He I $\lambda 10830$ line profiles into two types based on the shapes of the blueshifted wind absorption component. The first type has a relatively narrow and low wind absorption component (the upper right panel in Fig. 3). The second type has a wide and deep wind (P-Cygni like) absorption component which reaches the maximum velocity of $300-400 \mathrm{~km} \mathrm{~s}^{-1}$ (the lower right panel in Fig. 3). Edwards et al. (2006) suggested that 
the former is caused by a disk wind, and the star is viewed at a relatively high inclination angle at which a line of sight can intersect with the disk wind. On the other hand, the latter is cased by a stellar wind, and the star is viewed nearly pole-on.

To test the scenarios of Edwards et al. (2006) for the two distinctive wind absorption features, we have constructed the simple kinematic flow models around CTTSs. See Kurosawa et al. (2011) for the model descriptions. The first model consists of a disk wind and magnetospheric accretion funnels (the upper left panel in Fig. 3). The second model consists of a stellar wind and magnetospheric accretion funnels (the lower left panel in Fig. 3). The corresponding line profile models for He I $\lambda 10830$ are also shown in the same figure (the middle panels). Our disk wind + magnetosphere model reproduces not only the narrow blueshifted wind absorption component, but also the redshifted absorption component as seen in the observed He I $\lambda 10830$ line profile of the CTTS UY Aur. Note that the redshifted absorption component is caused by the infalling gas in the magnetospheric accretion funnel. Similarly, our stellar wind + magnetosphere model reproduces a rather wide and deep blueshifted absorption component as seen in the observed He I $\lambda 10830$ in the CTTS AS 353 A. Interestingly, the narrow wind absorption caused by the disk wind is present in the line profile only when the inclination angle of the system is an intermediate to a high value because of the geometry of the disk wind. Similarly, the wide and deep P-Cygni like wind absorption is present only at a very low inclination angle (near pol-on). Hence, our simple kinematic wind models confirms the earlier finding of Edwards et al. (2006) who suggested two different types of wind for the narrow and wide blueshifted absorption components seen in He I $\lambda 10830$. A similar analysis was also performed by Kwan et al. (2007) with simplified line emissivity and opacity in the winds.

In reality (as also suggested by Edwards et al. 2006), both types of the winds (a stellar and a disk winds) could coexist; however, it is likely that only one type of wind absorption component appears in a line profile because the line of sight to the stellar surface could intersect only one type of wind for a given viewing angle of the system.

\subsection{The Conical Wind Model}

To advance our understanding of the formation of the inner winds, we now apply our radiative transfer models to more realistic winds. For this purpose, we move our focus back to MHD simulation results. Recent MHD simulations by Romanova et al. (2009) have shown a new type of outflow configuration, so called "the conical wind" (the upper panel in Fig. 4), which is formed when a large scale stellar dipole magnetic field is compressed by the accretion disk into the X-wind (Shu et al. 1994) configuration. The outflows occur in a rather narrow conical shell, and the outflow speed of the matter is an order of the Keplerian rotation speed near the wind launching region. Interestingly, the conical wind model does not require the magnetospheric radius to be very close the corotation radius, unlike the X-wind model. Similar simulations with extended computational domain by Lii et al. (2012) have shown that the conical wind become collimated at large distance. The model was applied to the high mass-accretion rate FU Ori systems in which the wind can be strongly collimated (Königl et al. 2011).

We extended the original conical wind model of Romanova et al. (2009) to include a well-defined magnetospheric accretion funnel flow which is essential for modeling the strong optical and near-infrared emission lines in CTTSs. We achieved this by using a slightly stronger magnetic filed and a lower mass-accretion rate to reduced the compression of magnetosphere by the disk, hence to form a larger magnetospheric funnel flow. The resulting flow geometry is shown in Fig. 4 (the upper panel). We then used the density and velocity field from the MHD simulation, after it reached a semi-steady 

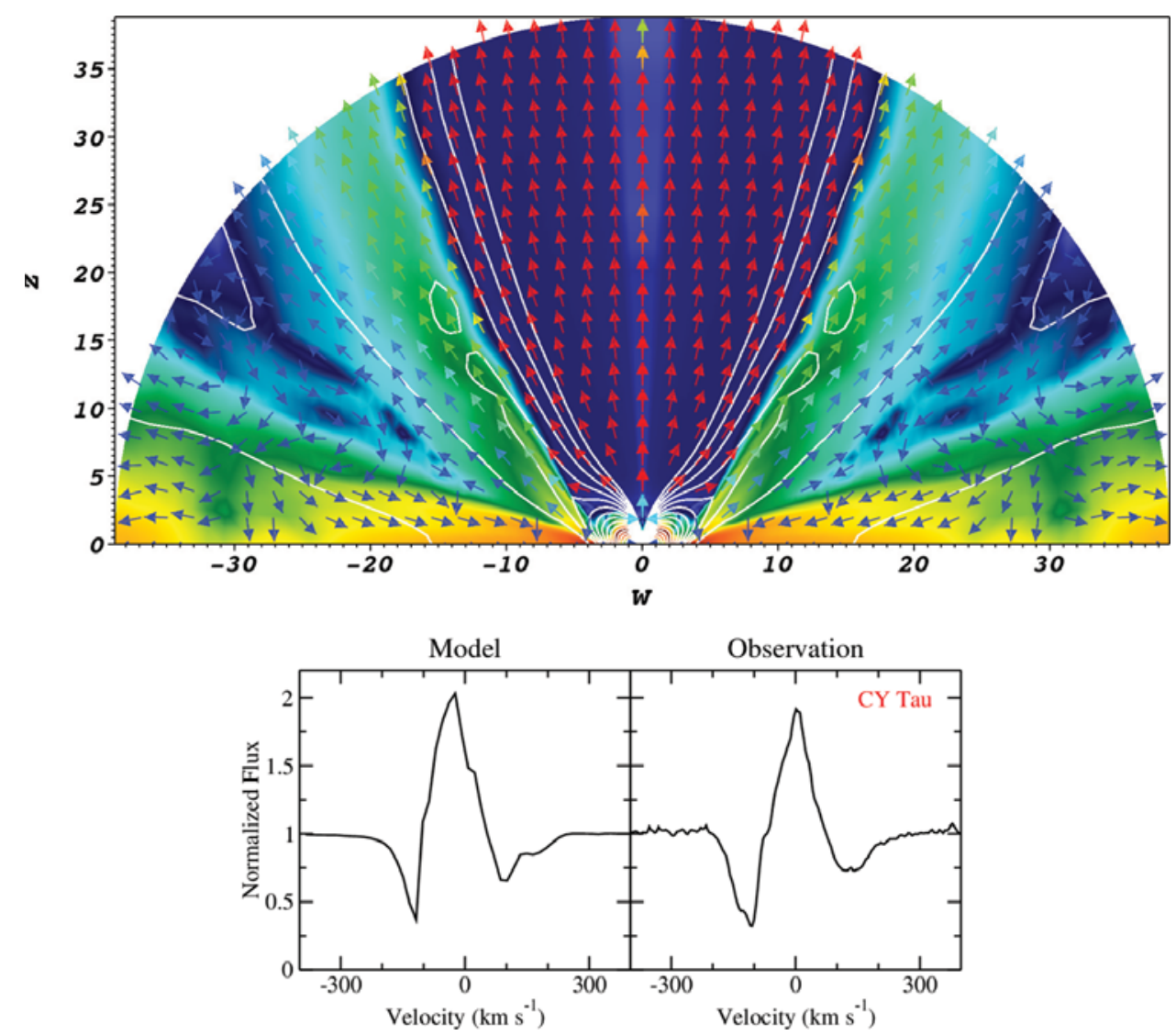

Figure 4. Summary of the conical wind model (Romanova et al. 2009) and a qualitative comparison of the model and observed He I $\lambda 10830$ profiles. Upper panel: the poloidal matter flux map is over-plotted with the poloidal component of the velocity (arrows). The solid contours represent the magnetic field lines. Lower panels: a qualitative comparison of the model profile of He I $\lambda 10830$ with the observation of the CTTS CY Tau (Edwards et al. 2006). The figures are from Kurosawa \& Romanova (2012)

state, in our radiative transfer calculations to predict hydrogen and helium line profiles. The line profiles were computed with various combinations of X-ray fluxes (important for photoionizing HeI), gas temperatures and inclination angles. A large variety of line profile morphology is found, and many of the model profiles are very similar to those found in observations. An example of He I $\lambda 10830$ line profile calculation along with the observation of the CTTS CY Tau (from Edwards et al. 2006) is shown in Fig. 4 (the lower panels). More examples can be found in Kurosawa \& Romanova (2012). As one can see in Fig. 4, the conical wind model can well reproduce the relatively narrow and low-velocity blueshifted wind absorption component in the observed line profile of He I $\lambda 10830$. It also reproduces the redshifted absorption component, which is caused by the infalling material in the accretion funnel flow. In summary, based on our line profile models, we found that the conical wind model is a very plausible outflow model for a system which shows a narrow and low-velocity blueshifted absorption component in He I $\lambda 10830$, and it is an important alternative model to a disk wind model which can also form the narrow and low-velocity blueshifted absorption component, as we have shown in Sec. 3.1. 


\section{Summary}

Recent observations suggest that the geometry of the magnetospheric accretion may significantly deviate from an axisymmetry for some of CTTSs. We have briefly reviewed the 3-D MHD simulations which considered the accretion onto CTTSs with inclined magnetic multipoles (e.g., a dipole and an octupole). Non-axisymmetric accretion funnel flows are naturally found in those simulations. Using the output from the simulations in our radiative transfer models, observable quantities such as line profiles and light curves are computed. The predictions can be readily compared with observations. While the periodic/regular variability behaviors seen in observations of CTTSs can be well reproduced by non-axisymmetric accretion models that are in a steady state (Sec. 2.1), the irregular variability behaviors seen in some of CTTSs could be explained by the magnetospheric accretion that is in unstable regime due the magnetic Rayleigh-Taylor (R-T) instability (Sec. 2.2). In addition to the irregular light curves and line variability, the unstable accretion due to the R-T instability would produce a variable but rather persistent redshifted absorption component in higher Balmer lines (e.g., $\mathrm{H} \gamma$ and $\mathrm{H} \delta$ ) and in some near-infrared hydrogen lines such as $\mathrm{Pa} \beta$ and $\mathrm{Br} \gamma$, due to the presence of many accretion streams caused by the instability.

We have also investigated the possible origins of the inner winds in CTTSs using the line profile models. Using the simple kinematic wind models in our radiative transfer models (Sec. 3.1), we confirmed the earlier finding by Edwards et al. (2006) who suggested that there are two types of inner winds of CTTSs. We confirmed that the relatively narrow and low-velocity blueshifted absorption component in He I $\lambda 10830$ can be reproduced by a disk wind model, and the wide and deep blueshifted absorption component in the same line can be reproduced by a stellar wind model. For the stellar wind component to be visible in the absorption component, the inclination angle of the system must be rather small. We have also tested the plausibility of the conical wind solution found in the recent MHD simulations by Romanova et al. (2009) by using the simulation results in our radiative transfer models (Sec. 3.2). We found the conical wind model can also reproduce the relatively narrow and low-velocity blueshifted absorption component in He I $\lambda 10830$ seen in some CTTSs. Finally, high resolution spectroscopic data and radiative transfer models are invaluable for testing different outflow and inflow scenarios since a direct imaging of the inner wind launching regions is still unattainable for most of the CTTSs.

\section{Acknowledgment}

We thank the conference organizers for the excellent meeting. RK thank Silvia Alencar, Suzan Edwards and Tim Harries for their support and discussions. Resources supporting this work were provided by the NASA High-End Computing (HEC) Program through the NASA Advanced Supercomputing (NAS) Division at Ames Research Center and the NASA Center for Computational Sciences (NCCS) at Goddard Space Flight Center. The research was supported by NASA grants NNX11AF33G and NSF grant AST-1211318.

\section{References}

Alencar, S. H. P. \& Basri, G. 2000, AJ, 119, 1881

Alencar, S. H. P., Bouvier, J., Walter, F. M., Dougados, C., Donati, J.-F., Kurosawa, R., Romanova, M., Bonfils, X., Lima, G. H. R. A., Massaro, S., Ibrahimov, M., \& Poretti, E. 2012, A\&A A, 541, A116

Alencar, S. H. P., Teixeira, P. S., Guimarães, M. M., McGinnis, P. T., Gameiro, J. F., Bouvier, J., Aigrain, S., Flaccomio, E., \& Favata, F. 2010, A\& $A$, 519, A88 
Ardila, D. R., Basri, G., Walter, F. M., Valenti, J. A., \& Johns-Krull, C. M. 2002, ApJ, 567, 1013

Arons, J. \& Lea, S. M. 1976, ApJ, 207, 914

Calvet, N. \& Gullbring, E. 1998, ApJ, 509, 802

Camenzind, M. 1990, in Reviews in Modern Astronomy, Vol. 3, Reviews in Modern Astronomy, ed. G. Klare, 234

Donati, J.-F., Bouvier, J., Walter, F. M., Gregory, S. G., Skelly, M. B., Hussain, G. A. J., Flaccomio, E., Argiroffi, C., Grankin, K. N., Jardine, M. M., Ménard, F., Dougados, C., \& Romanova, M. M. 2011, MNRAS, 417, 472

Donati, J.-F., Jardine, M. M., Gregory, S. G., Petit, P., Bouvier, J., Dougados, C., Ménard, F., Collier Cameron, A., Harries, T. J., Jeffers, S. V., \& Paletou, F. 2007, MNRAS, 380, 1297

Edwards, S., Fischer, W., Hillenbrand, L., \& Kwan, J. 2006, ApJ, 646, 319

Edwards, S., Fischer, W., Kwan, J., Hillenbrand, L., \& Dupree, A. K. 2003, ApJ, 599, L41

Ferreira, J., Dougados, C., \& Cabrit, S. 2006, A\& $\&$, 453, 785

Ghosh, P., Pethick, C. J., \& Lamb, F. K. 1977, ApJ, 217, 578

Gregory, S. G., Matt, S. P., Donati, J.-F., \& Jardine, M. 2008, MNRAS, 389, 1839

Harries, T. J. 2000, MNRAS, 315, 722

Hartmann, L., Hewett, R., \& Calvet, N. 1994, ApJ, 426, 669

Herbst, W., Herbst, D. K., Grossman, E. J., \& Weinstein, D. 1994, AJ, 108, 1906

Hussain, G. A. J., Collier Cameron, A., Jardine, M. M., Dunstone, N., Ramirez Velez, J., Stempels, H. C., Donati, J.-F., Semel, M., Aulanier, G., Harries, T., Bouvier, J., Dougados, C., Ferreira, J., Carter, B. D., \& Lawson, W. A. 2009, MNRAS, 398, 189

Jardine, M. M., Gregory, S. G., \& Donati, J.-F. 2008, MNRAS, 386, 688

Johns, C. M. \& Basri, G. 1995, ApJ, 449, 341

Koenigl, A. 1991, ApJ, 370, L39

Königl, A., Romanova, M. M., \& Lovelace, R. V. E. 2011, MNRAS, 416, 757

Kulkarni, A. K. \& Romanova, M. M. 2008, MNRAS, 386, 673

Kulkarni, A. K. \& Romanova, M. M. 2009, MNRAS, 398, 701

Kurosawa, R., Harries, T. J., \& Symington, N. H. 2005, MNRAS, 358, 671

Kurosawa, R., Harries, T. J., \& Symington, N. H. 2006, MNRAS, 370, 580

Kurosawa, R. \& Romanova, M. M. 2012, MNRAS, 426, 2901

Kurosawa, R. \& Romanova, M. M. 2013, MNRAS, 431, 2673

Kurosawa, R., Romanova, M. M., \& Harries, T. J. 2008, MNRAS, 385, 1931

Kurosawa, R., Romanova, M. M., \& Harries, T. J. 2011, MNRAS, 416, 2623

Kwan, J., Edwards, S., \& Fischer, W. 2007, ApJ, 657, 897

Li, L.-X. \& Narayan, R. 2004, ApJ, 601, 414

Lii, P., Romanova, M., \& Lovelace, R. 2012, MNRAS, 420, 2020

Long, M., Romanova, M. M., Kulkarni, A. K., \& Donati, J.-F. 2011, MNRAS, 413, 1061

Muzerolle, J., Calvet, N., \& Hartmann, L. 2001, ApJ, 550, 944

Petrov, P. P., Gullbring, E., Ilyin, I., Gahm, G. F., Tuominen, I., Hackman, T., \& Loden, K. 1996, A\&̈A, 314, 821

Reipurth, B., Pedrosa, A., \& Lago, M. T. V. T. 1996, A\&AS, 120, 229

Romanova, M. M., Kulkarni, A. K., \& Lovelace, R. V. E. 2008, ApJ, 673, L171

Romanova, M. M., Long, M., Lamb, F. K., Kulkarni, A. K., \& Donati, J.-F. 2011, MNRAS, 411, 915

Romanova, M. M., Ustyugova, G. V., Koldoba, A. V., \& Lovelace, R. V. E. 2004, ApJ, 610, 920

Romanova, M. M., Ustyugova, G. V., Koldoba, A. V., \& Lovelace, R. V. E. 2009, MNRAS, 399, 1802

Romanova, M. M., Ustyugova, G. V., Koldoba, A. V., Wick, J. V., \& Lovelace, R. V. E. 2003, ApJ, 595, 1009

Rucinski, S. M., Matthews, J. M., Kuschnig, R., Pojmański, G., Rowe, J., Guenther, D. B., Moffat, A. F. J., Sasselov, D., Walker, G. A. H., \& Weiss, W. W. 2008, MNRAS, 391, 1913 
Shu, F., Najita, J., Ostriker, E., Wilkin, F., Ruden, S., \& Lizano, S. 1994, ApJ, 429, 781

Spruit, H. C. \& Taam, R. E. 1993, ApJ, 402, 593

Symington, N. H., Harries, T. J., \& Kurosawa, R. 2005, MNRAS, 356, 1489

Takami, M., Chrysostomou, A., Bailey, J., Gledhill, T. M., Tamura, M., \& Terada, H. 2002, ApJ, 568, L53 